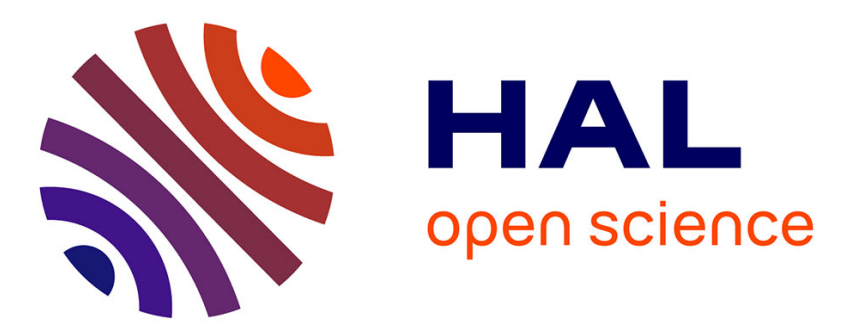

\title{
Phase-locking modes in a bidimensional network of coupled water jets
}

S. Houard, F. Daviaud, P. Bergé

\section{To cite this version:}

S. Houard, F. Daviaud, P. Bergé. Phase-locking modes in a bidimensional network of coupled water jets. Physica D: Nonlinear Phenomena, 1996, 99, pp.318-338. 10.1016/S0167-2789(96)00140-6 . cea01373968

\section{HAL Id: cea-01373968 https://hal-cea.archives-ouvertes.fr/cea-01373968}

Submitted on 29 Sep 2016

HAL is a multi-disciplinary open access archive for the deposit and dissemination of scientific research documents, whether they are published or not. The documents may come from teaching and research institutions in France or abroad, or from public or private research centers.
L'archive ouverte pluridisciplinaire HAL, est destinée au dépôt et à la diffusion de documents scientifiques de niveau recherche, publiés ou non, émanant des établissements d'enseignement et de recherche français ou étrangers, des laboratoires publics ou privés. 


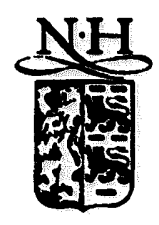

ELSEVIER

\title{
Phase-locking modes in a bidimensional network of coupled water jets
}

\author{
S. Houard *, F. Daviaud, P. Bergé \\ Service de Physique de l'Etat Condensé, CEA/Saclay, F-91191 Gif-sur-Yvette Cédex, France
}

Received 31 July 1995; revised 1 May 1996; accepted 1 May 1996

Communicated by Y. Kuramoto

\begin{abstract}
In this paper, we investigate the dynamics of a bidimensional network of coupled water jets impinging from below on a water/air interface. For each jet, a transition is observed at a critical flow rate value for which the surface bump at the vertical of the jet starts oscillating at a well-defined frequency. We infer that this oscillatory mode is the materialization at the surface of a helical instability of the submerged laminar jet. When coupled together, the bidimensional network of oscillators exhibits monoperiodic collective modes whose spatial arrangements are similar to those encountered in crystals. A collection of phase-locking modes is observed for each geometry, and stability diagrams are constructed. Analysis of the coupling between the jets reveals a long distance coupling through surface waves. A tuning criterion is proposed to explain the bifurcation from one mode to another. Finally, the symmetries of the system are investigated using two different systematic schemes. The predictions are compared with the observations and some features of the particular topology of phase-locking modes are explained.
\end{abstract}

PACS: 0545; 4755C; 0220

Keyworts: Jet; Helical instability; Phase-locking mode; Bifurcation; Symmetry

\section{Introduction}

The study of the collective dynamics of systems of coupled oscillators has received considerable interest in the last few years. This results from the wide variety of problems it can be connected to: from the study of collective chaos in theoretical physics [1-7], to the study of the cooperative synchronization phenomenon and of biological rhythms in biology [8-14]. Indeed, mutual synchronization occurs very commonly in populations of biological oscillators: congregated fireflies flashing in synchrony at night $[8,12]$, pacemaker cells of the heart working in unison [11] and women having

\footnotetext{
*Corresponding author.
}

their menstrual periods in synchrony [9] are striking examples of cooperative synchronization.

A first mathematical approach of the phenomenon of mutual synchronization was proposed by Winfree [15]. He considered populations of weakly coupled oscillators, whose individual limit cycles were not perturbed, so that only phase variations had to be considered. He discovered that synchronization occurs above some coupling strength threshold value. His phase model was reformulated by Kuramoto [16]. Three different regimes were observed, depending on the coupling strength relative to the frequency bandwidth of the population of oscillators [4]: (i) incoherence, where the oscillators beat at different frequencies, (ii) phase-locking modes, including the 
trivial mode of synchrony, where the oscillators beat at the same frequency, but present constant phaseshift relative to each other, (iii) partial locking, a state intermediate between the latter two. The most striking example of phase-locking mode in nature is in the locomotion of animals $[14,17]$, where the different gates of quadrupeds correspond to phase-locking modes of different symmetry.

As a further step, models with stronger coupling between oscillators were considered, allowing amplitude variations of limit cycles. This led to the discovery of unsteady collective modes, such as collective chaos, that were not observable with the earlier phase models [4]. The collective behaviour of networks of coupled oscillators is now investigated by many physicists, using the formalism of discretized complex Ginzburg-Landau equations [6-7]. Among other things, the chaotic dynamics in such systems is expected to exhibit characters intermediate between low-dimensional chaos and spatio-temporal chaos [7].

Parallel to this intensive numerical work, the spatio-temporal behaviour of experimental systems of coupled oscillators has been investigated too, involving Josephson-junction series arrays [18] or 2D lattices of coupled units such as vortices [19] or electronic devices [20]. In this paper, we propose to study the collective dynamics of a 2D network of coupled oscillators using an original experimental device: co-flowing water jets impinging (from below) on a water/air interface.

Although many studies have been devoted to the problem of a jet impinging on a flat solid surface [2123], few have been devoted to the imf igement of a submerged jet on a water/air interface, and if so $[24,25]$, for a jet flow parallel to the surface only. The present experimental set-up, namely a jet impingement normal to the water/air interface has, to our knowledge, not been investigated yet. Thus we propose to study the collective dynamics of bidimensional networks of such co-flowing jets. Note that Villermaux and Hopfinger [26] studied large-scale instabilities in a bidimensional network of co-flowing air jets, but no interface was present.

First, the experimental device is presented. Then the case of a single jet is considered: above some flow rate threshold value, a single water jet exhibits a monoperiodic oscillatory mode. The characteristics and mechanism of transition are studied in detail. Then, the dynamics of two coupled jets, a line of jets and finally bidimensional lattices of such co-flowing water jets are investigated, using perforated plates of different geometries and solidities. Collective phase-locking modes are observed, whose topology is commented upon. For each set of plate parameters, diagrams of stability are constructed which are used to investigate the coupling mechanism between jets, as well as the criteria of transition from one phase-locking mode to another. In a last step, considerations of symmetry are used to highlight the particular topology of phaselocking modes, with two different approaches. The first approach is specific to the present experiment, and is derived from the empirical procedure which was adopted for observations, while the second is more generic, and uses group theory.

\section{Experimental set-up}

In the present experiment, we study the collective dynamics of a bidimensional network of coupled water jets. To generate the jets, a plexiglass tranquilization chamber is used (Fig. 1). The water comes in through a thin cylinder and penetrates in the chamber through two $3 \mathrm{~mm}$ holes perforated at its end. The turbulent flow is then homogenized using a grid and a $5 \mathrm{~cm}$ thick dense sponge. A sponge was preferred to straws because the vertical size of the whole apparatus had to be limited. The flow then comes through a $6 \mathrm{~mm}$ thick perforated plate, generating co-flowing water jets. Note that in a first prototype, the jets issued from $30 \mathrm{~mm}$ long pipes instead, in order to facilitate the backward recirculation of the flow. However, since no quantitative change in the collective dynamics was observed, we opted for the more convenient device of Fig. 1. Indeed, since the perforated plates are removable, various geometries and plate parameters can be tested. Three geometries have been investigated: square, hexagonal (honeycomb) and triangular, with different plate parameters for each of them (Table 1). The square, hexagonal and triangular meshes of the 


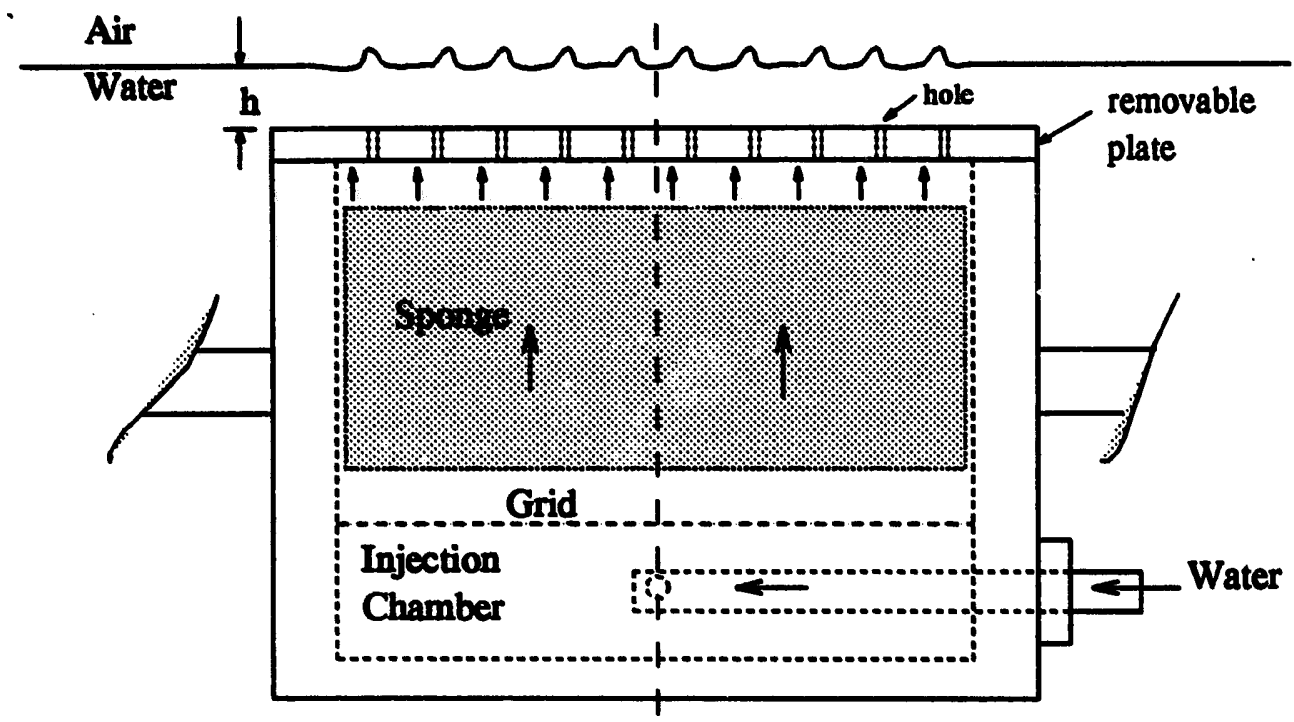

Fig. 1. Experimental device. A tranquilization chamber is used to homogenize the turbulent incoming flow. Then, the flow passes through a removable perforated plate, generating co-flowing water jets.

Table 1

List of plate parameters

Network mesh

\begin{tabular}{|c|c|c|c|}
\hline Jet diameter & $\square$ & 3 & $\circledast$ \\
\hline $\begin{array}{l}\phi=1.44 \mathrm{~mm} \\
\phi=1.0 \mathrm{~mm} \\
\phi=0.5 \mathrm{~mm}\end{array}$ & $\begin{array}{l}N=89, d=8 \mathrm{~mm} \\
N=57, d=12,6,4 \mathrm{~mm} \\
N=57, d=12,6 \mathrm{~mm}\end{array}$ & $N=46, d=12,8 \mathrm{~mm}$ & $N=68, d=12,8 \mathrm{~mm}$ \\
\hline
\end{tabular}

Note: $N$ - the number of jets of the network; $d$ - the distance between nearest neighbours.

network are represented by the corresponding symbols in Table $1 . N$ is the number of jets of the network, while $d$ is the distance between nearest neighbours. Except for the square geometry, only $1.0 \mathrm{~mm}$ holes have been used.

The plexiglass tranquilization chamber is immersed in water in a $0.4 \mathrm{~m}$ deep, $0.4 \mathrm{~m}$ wide and $0.7 \mathrm{~m}$ long plexiglass water tank. The chamber can be positioned vertically using a micropositioner, so that the depth of the jets below the free-surface can be adjusted. The water is pumped at the bottom of the tank using a centrifugal pump, and reinjected at the bottom of the tranquilization chamber (Fig. 1). The free surface level is thus always constant in the tank. Moreover, the water tank itself is large enough for the pumping of the water not to create perturbations in the hydrodynamics of the jets. A flowmeter is used to control the total flow rate through the perforated plate. The homogeneity of the flow throughout the whole network of jets has been verified at low flow rate values, using an ombroscopic method. The homogeneity of the 2D light reflection patterns obtained, along with the great sensitivity of the method, allowed us to conclude that the flow rate was homogeneous throughout the network.

The presence of a water/air interface allows a bidimensional visualization of the system. In the counterpart, it introduces complexity in the physical processes, since the water jets are impinging on a deformable surface. Actually, the interface is part of the physical system and reveals to be crucial, both for the development of the oscillatory phenomenon which is observed and for the coupling between the jets.

The dynamics of the system is expected to depend both on (i) physical parameters governing the dynamics of a jet: the flow $F$ (or the velocity $F / S$ ) at the jet nozzle and the depth $h$ of the jet nozzle 

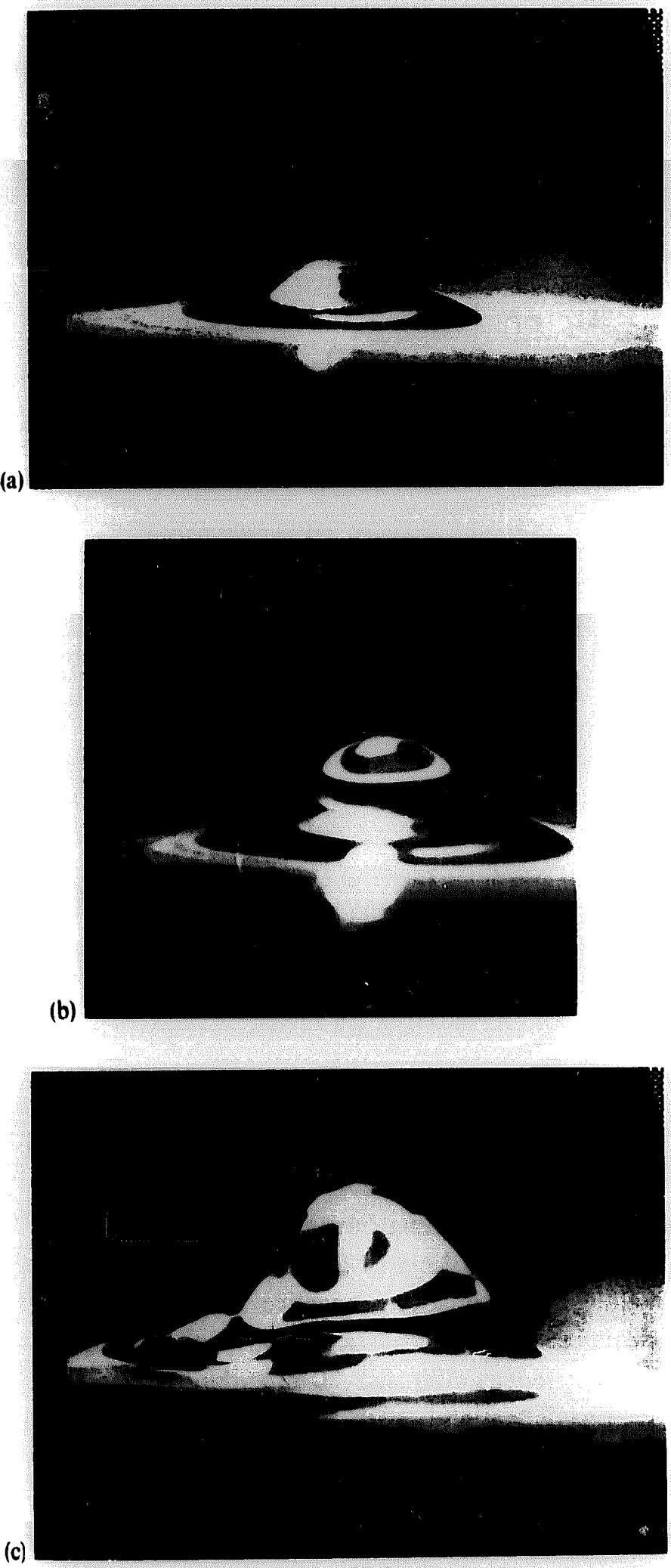

below the free-surface; (ii) geometrical parameters: the geometry of the network, the distance $d$ between nearest neighbours in the lattice, the diameter $\phi$ of the holes and the number $N$ of jets in the network; (d)

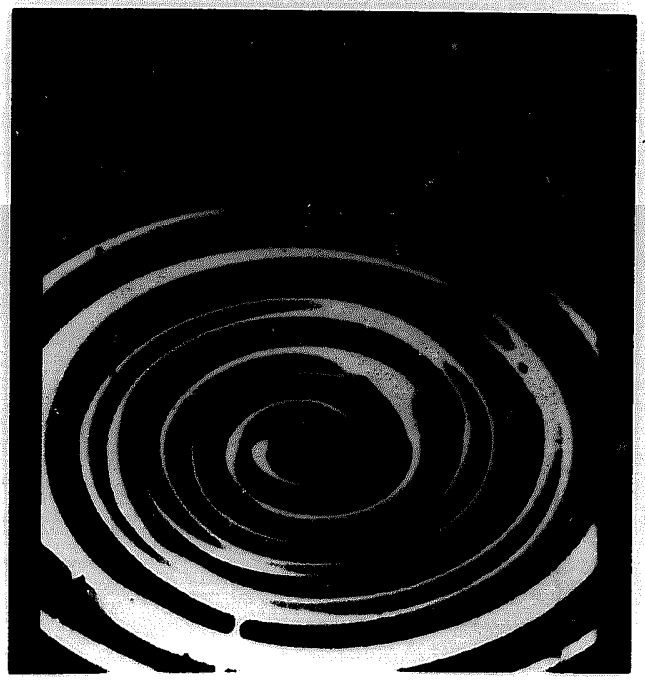

Fig. 2. Surface deformation at the vertical of a jet, for increasing values of the adimensional parameter $\varepsilon=\left(F-F_{\mathrm{c}}\right) / F_{\mathrm{c}}$. ( $\phi=1.44 \mathrm{~mm} ; h=10 \mathrm{~mm}$ ): (a) $\varepsilon=-0.2$; (b) $\varepsilon=-0.1$; (c) $\varepsilon=0$ : a transition is observed where the bump starts oscillating at a high eigenfrequency; (d) surface waves generated by the oscillatory motion of the bump. In both panels (c) and (d), stroboscopic lighting is used to visualize the phenomenon. In panels (a)-(c), the zoom magnification factor is 20 .

(iii) characteristics of the fluid: surface tension and viscosity.

\section{Oscillatory mode for a single jet}

\subsection{Observations}

The impingement of the jet on the water/air interface induces an upward deflection of the interface at the vertical of the jet. The height of the deflection increases with increasing flow rate at the nozzle exit. Fig. 2 illustrates the evolution of the free-surface deformation as the flow rate increases. First, only a small and smooth swell is visible (Fig. 2(a)); then a higher bump bursts at the top of the small swell (Fig. 2(b)). Then this bump grows up to a critical height where it starts oscillating (Fig. 2(c)) in a circular motion at the same time as surface waves are generated (Fig. 2(d)). The height of the bump at the threshold is of a few millimeters, but depends both on the diameter $\phi$ of the jet and on the depth $h$ of the jet nozzle. The eigenfrequency of the oscillation is larger than the standard video frequency, and a 


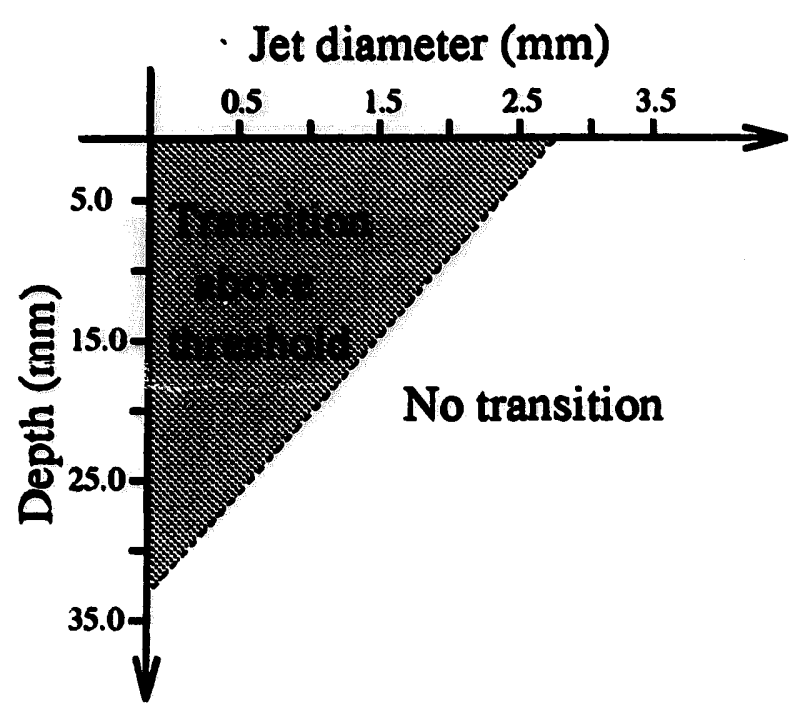

Fig. 3. Field of existence of the transition. Note that the transition to the oscillatory mode is no longer observed for jet di-' ameters exceeding the capillary length of water $\left(l_{c}=2.0 \mathrm{~mm}\right)$.

stroboscopic lighting is thus necessary to visualize the phenomenon (Figs. 2(c) and (d)).

The oscillatory transition is observed for very narrow ranges of parameters. It is no longer observed for jet diameters larger than $2.5 \mathrm{~mm}$, nor is it observed for too large depths $h$. Actually, the smaller the diamter of the jet, the deeper the transition can be observed: from a maximum depth of $1.5 \mathrm{~cm}$ for a $2.0 \mathrm{~mm}$ diameter jet to a maximum depth of $3 \mathrm{~cm}$ for a $0.5 \mathrm{~mm}$ diameter jet. This is summarized, qualitatively, in Fig. 3, where the shaded area represents the existence field of the transition: in this area, the oscillatory mode appears above a flow rate threshold value depending both on the diameter $\phi$ and on the depth $h$ of the jet.

\subsection{Measurements}

\subsubsection{Measurements at the threshold}

Using a photodiode (placed above a given point of the free-surface) and a large spectrum lighting source, the time dependence of the surface deformation has been analysed using a spectrum analyser. Just above the threshold, the spectrum displays a fundamental mode and harmonics (cf. [27]). The dependence of the frequency of the fundamental mode with the depth $\boldsymbol{h}$ and the diameter $\phi$ of the jet nozzle has been ex-
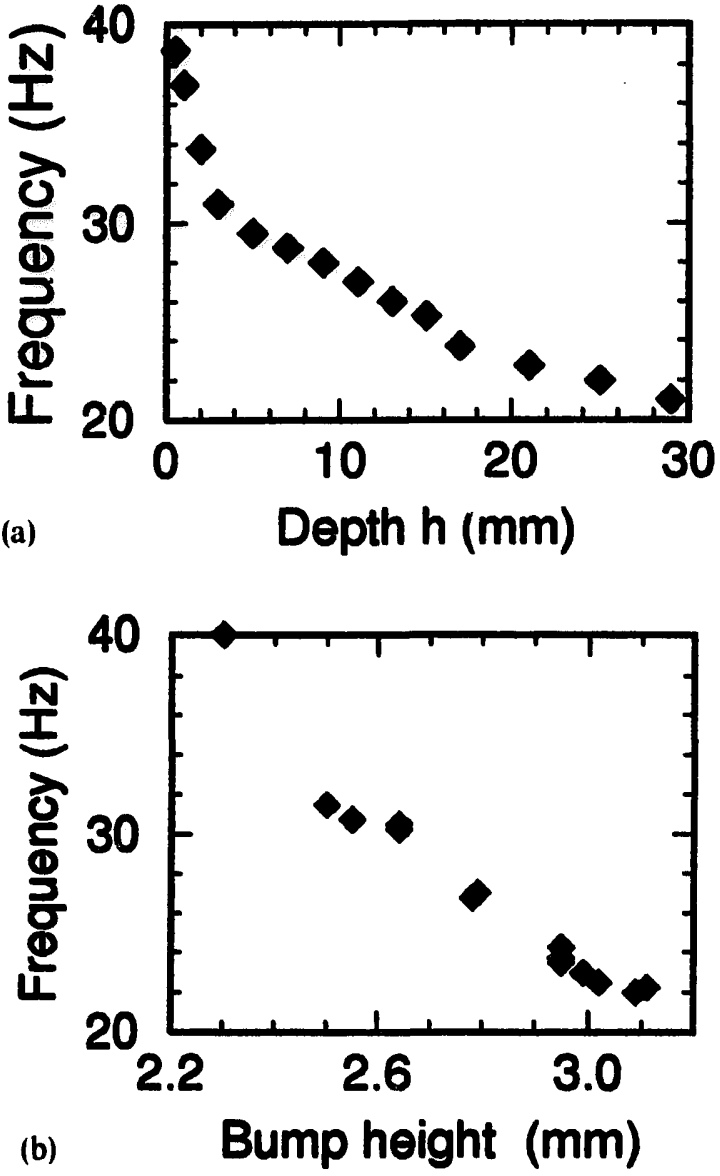

Fig. 4. (a) Variations of the eigenfrequency of the oscillatory mode (at the threshold) versus depth, $\phi=1.44 \mathrm{~mm}$. (b) Correlation between the eigenfrequency of the mode and the bump height (at the threshold), $\phi=1.44 \mathrm{~mm}$.

amined closely. Fig. 4(a) shows the variation of the frequency of the oscillatery mode (at the threshold) versus the depth $h$ for a $1.44 \mathrm{~mm}$ diameter jet. The frequency is a decreasing function of depth. Three linear trends can be distinguished: for very shallow depths, below $5 \mathrm{~mm}$, a strong negative slope is observed; then, between 5 and $20 \mathrm{~mm}$, the slope is moderate; finally, between 20 and $30 \mathrm{~mm}$, the frequency decreases only slightly with depth. For the same depths, the height of the bump (at the threshold) has also been measured. When the frequency is plotted versus the height of the bump (Fig. 4(b)), a linear trend is observed, and the frequency decreases with increasing height. Note that the frequency (at the threshold) decreases from 40 to $20 \mathrm{~Hz}$, while the bump height (at the threshold) increases from 2.2 to $3.1 \mathrm{~mm}$, when the depth $h$ is increased from 1 to $30 \mathrm{~mm}$ (Fig. 4(b)). 
The dependence of the frequency upon the diameter of the jet has also been examined. For a depth of $10 \mathrm{~mm}$, the frequency at the threshold is 36,27 and $20 \mathrm{~Hz}$, respectively for jet diameters of $1.0,1.44$ and $2.0 \mathrm{~mm}$ : the frequency decreases when the diameter of the jet increases.

\subsubsection{Measurements above the threshold (at a fixed depth)}

When the flow is increased above the threshold, the fundamental peak is enlarged and the harmonics disappear, buried in the background noise: the sharpness of the monoperiodic mode decreases as the spreading from the threshold increases. Indeed, the motion of the bump appears to be more complex: the oscillation in the horizontal plane is contaminated by the vertical gravitational collapses of the bump, hence the enlargement of the peak. At the end, the spectrum has become large and the jet is in the well-known fountain regime. In Fig. $5(a)$, the frequency is plotted versus the relative spreading $\varepsilon=\left(F-F_{\mathrm{c}}\right) / F_{\mathrm{c}}$ from the flow rate threshold $F_{\mathrm{c}}$, at depths of 2,5 and $10 \mathrm{~mm}$, for a jet diameter of $1.44 \mathrm{~mm}$. For a given depth, two different symbols are used to distinguish the oscillatory regime (diamonds) and the fountain regime (squares). Note that the frequency of the oscillatory mode decreases linearly as $\varepsilon$ increases. The slope is the same for depths of 5 and $10 \mathrm{~mm}$, but is slightly different for a depth of $2 \mathrm{~mm}$. In Fig. 5(b), the corresponding bump height is plotted versus $\varepsilon$ for depths of 5 and $10 \mathrm{~mm}$. The symbols are the same as in Fig. 5(a), except that a third symbol (circles) is used for negative values of $\varepsilon$, corresponding to the case of Figs. 2(a) and (b). In the oscillatory regime, the height of the bump increases linearly as $\varepsilon$ increases. When plotted together, the frequency and the bump height still display a negative linear tendency as in Fig. 4(b), but the slope is smaller. The difference in slope may result from the vertic:l fluctuations of the bump observed above the threshold: the height which is indeed measured is the maximum height reached by the bump. The physical link with the frequency is thus perhaps not as direct as for the measures at the threshold.
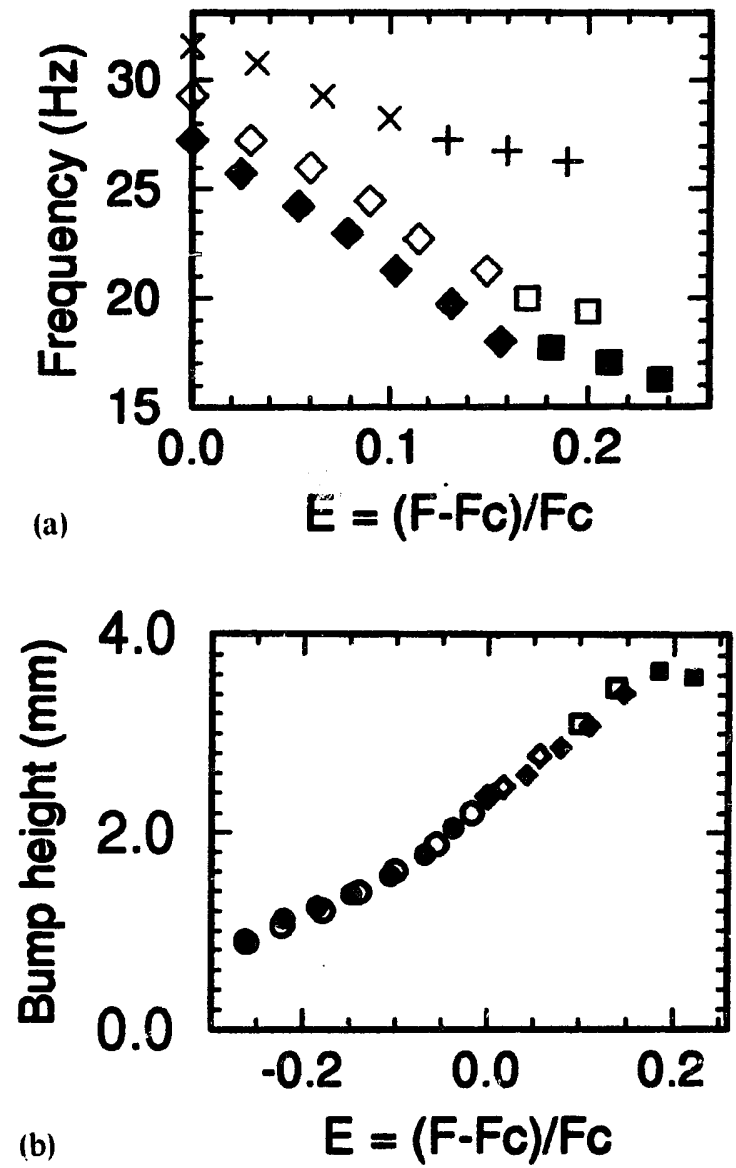

Fig. 5. (a) Variations of the eigenfrequency of the oscillatory mode for increasing flow rate values above the threshold. $\phi=1.44 \mathrm{~mm} ; x+: h=2 \mathrm{~mm} ; \Delta \square: h=5 \mathrm{~mm} ; \bullet$ $h=10 \mathrm{~mm}$. (b) Variations of the bump height for increasing flow rate values above the threshold. $\phi=1.44 \mathrm{~mm}$. Non-filled symbols: $h=5 \mathrm{~mm}$; filled symbols: $h=10 \mathrm{~mm}$. $\circ \bullet: \varepsilon<0$, no motion; $\diamond: \varepsilon>0$, oscillatory mode; $\square$ : $\varepsilon>0$, fountain regime.

To summarize:

- above some flow rate threshold value, the bump at the vertical of the jet exhibits a monoperiodic oscillatory mode,

- at the same time, surface waves with a spiral geometry are generated,

- at the threshold, the frequncy of the mode decreases with increasing depth or with increasing diameter of the jet. The decrease is more pronounced at shallow depths,

- when the flow rate is increased above the threshold, the bump height increases while both the sharpness of the mode and its frequency decrease,

- the frequency decreases linearly with increasing bump height. 


\subsection{Jet instability}

At this stage, we would like to give some highlights on the possible origin and mechanism of the transition. The existence of a water/air interface is likely to play a crucial role in the oscillatory phenomenon which is observed. Indeed, the role of the capillary forces is pointed out (Fig. 3), by the fact that the transition is no longer observed for jet diameters larger than the capillary length of water $\left(l_{c}=2 \mathrm{~mm}[26]\right)$. The role of surface tension has been verified by adding drops of liquid soap: the threshold of the transition is then reduced significantly, whereas the frequency of the oscillation is only slightly modified. Below the threshold, a steady deformation of the surface is observed (Figs. 2(a) and (b)), which results from the vertical balance of the following forces: gravity, which tends to reduce the height of the bump; surface tension, which tends to minimize the surface of the bump; and the thrusting force of the jet, which tends to increase the height of the bump, which is interpreted as an entrainment motion initiated by a jet helical instability.

At the threshold, the surface bump undergoes a circular motion (Fig. 2(c)), generating surface ripples with a spiral pattern (Fig. 2(b)). The spiral pattern indicates that the source radiating the ripples is rotating at the frequency of the bump motion itself. Since laminar jets display helical instabilities that would well explain the particular geometry of the waves at the surface, we think that the instability we observe originates in the submerged water jet beneath the bump.

Few studies have been devoted to instabilities in a laminar jet. Batchelor and Gill [29], using a linear stability analysis in an inviscid fluid, showed that the helical and sinuous modes were the preferred modes of instability for a laminar jet. In an early dye experiment, Reynolds [30] observed sinuous undulations in a naturally excited liquid jet for a Reynolds number between 10 and 300. Both experimental [31-33] and theoretical $[31,34,35]$ studies have since confirmed the existence and predominance of helical instabilities in such laminar jets. In the present experiment, the Reynolds number $R_{e}=u \phi / v$ of the jets (where $u=F / S$ is the ratio of the flow rate over the nozzle surface, and $\phi$ the diameter of the jet) remains moderate $-300-600$ at the transition threshold - but is large enough for instabilities to be able to develop [36]. However, the development of instabilities in unstable jets is highly dependent on the velocity profile at the nozzle exit of the jet. In our experiment, the moderate Reynolds number $R e$ of the jets, along with the large nozzle length to nozzle diameter ratio $r$ of the jets $(r=6$ with perforated plates, $r=20$ with the early prototype with pipes), should make this profile rather smooth, if not that of a laminar jet exactly.

As a conclusion, we think that the oscillatory motion of the surface bump is a materialization of the helical instabilities which develop in the submerged water jet. Since a dynamic upward pressure is needed to maintain the surface bump to a given height, and since no variation of the bump height is observed during its oscillatory motion (at the threshold at least), it shiows that the surface bump just follows the rotary motion of the jet column in perfect synchrony. However, the existence of a correlation between the surface bump height and the eigenfrequency of the oscillation (Section 3.2) indicates that the surface, which is the upper limit of the oscillating jet column, may control the eigenfrequency of the instability through the size of the bump. Indeed, considering the bump like the mass of an inverted pendulum would well explain the decrease of frequency observed for increasing bump height (or increasing flow rate).

Note that a sinuous mode is also observed for rather shallow depths (a few millimeters) and for flow rates just above the threshold. The bump then describes a rectilinear motion and hemi-circular concentric ripples are generated. However, when the depth is increased, or when the flow rate above the threshold is increased, only the helical mode is observed, in agreement with previous observations or stability analysis [29,31].

\section{Collective modes}

\section{I. Two coupled jets and line of coupled jets}

Above the flow rate threshold value, each jet is an oscillator whose eigenfrequency depends both 
on the depth $h$ and on the flow rate $F$. To begin with, we conducted simple experiments with two coupled oscillating jets. The influence of the depth $h$, the flow rate $F$ and the distance $d$ between the jets was investigated. When $d$ is smaller or equal to $12 \mathrm{~mm}$, the jets oscillate at the same frequency and are either in phase (acoustic mode) or $\pi$ phase-shifted (optical mode). Transitions are observed between the two modes with varying flow rate or depth. When the distance $d$ between the jets is increased, the jets begin to display incoherency, oscillating at two distinct frequencies. However, the difference $\Delta f$ depends on the depth of the jets below the surface. For instance, for $d=24 \mathrm{~mm}$, we measured $\Delta f$ for different depths at the flow rate threshold of the instability, we obtained $\Delta f=4 \mathrm{~Hz}$ at $h=2 \mathrm{inm} ; \Delta f=2.5 \mathrm{~Hz}$ at $h=5 \mathrm{~mm} ; \Delta f=$ $1.5 \mathrm{~Hz}$ at $h=10 \mathrm{~mm}$ and $\Delta f=0$ (synchrony) for depths larger than $13 \mathrm{~mm}$. One should keep in mind that the deeper the jets below the surface, the larger the bumps at the surface at the critical flow rate, and the stronger the surface waves. If, as will be developed in a following section, we think that the oscillators couple through the surface waves radiating from the bumps, it should not be surprising that synchrony is recovered for large depths. It is thus uneasy to determine a unique critical distance of interaction between the jets. Moreover, for a given depth $h$, the critical distance depends also on the couple of jets chosen. For instance, for one couple, incoherency appeared at $d=48 \mathrm{~mm}$, whereas synchrony still prevailed for another couple. This may result from little differences in diameter when the holes are machined. The "population" of oscillating jets could then present a frequency bandwidth $\Delta f$ depending on the quality of the machining.

Systems consisting of lines of jets have also been investigated. Acoustic and optical modes similar to those of systems of lines of vortices [19] were observed, with transitions between the two modes when the flow rate or the depth are varied. It should be noted that when increasing the flow rate, the instabilities usually appear first for the two jets situated at the end of the line.

\subsection{Bidimensional networks}

\subsubsection{Description of the modes}

Let us now explore the collective dynamics of bidimensional networks of such oscillators. To do that, a set of removable plexiglass plates has been machined, including three different geometries, and for each of them different sets of plate parameters (Table 1).

When coupled together, the jets always remain synchronized at the same frequency and oscillate with the same amplitude. Note that unlike in the two-jet system, and because of the necessary confinement of the experiment, the first neighbours of any jet are now always closer than $12 \mathrm{~mm}$ (Table 1). The collective modes display a monoperiodic time dependence simply characterized by its eigenfrequency. The spatial organization of the system is dominated by phase-locking phenomena: the initial network of jets subdivides into several sublattices of in-phase jets. To determine the spatial organization of the system, a stroboscopic lighting is used, at a frequency close to that of the eigenfrequency, and the residual motion of the network of bumps is observed. A grazing or vertical (using a beam-splitter plate) stroboscopic lighting is used, and a video recorder is placed at the vertical of the system.

In the helical instability mode, each oscillator, or bump, is sharacterized by its direction of rotation and by its phase shift relative to other bumps. To determine the latter, note that since the source of the ripples is rotating, the initiating point of the spiral pattern (Fig. 2(d)) can be identified easily at every instant. To identify the different collective modes, the following procedure is adopted: a given jet is taken as a reference, and the comparative state of its nearest neighbours is observed (comparative direction of the helix and phase-shift). This empirical procedure proved to be the most accurate since all collective modes show invariance by translation properties.

For each network geometry, different sets of plate parameters have been tested (Table 1), and a few collective modes have been identified. For each mode, a name has been given, specific to the particular arrangement of the bumps. All the modes that have been 


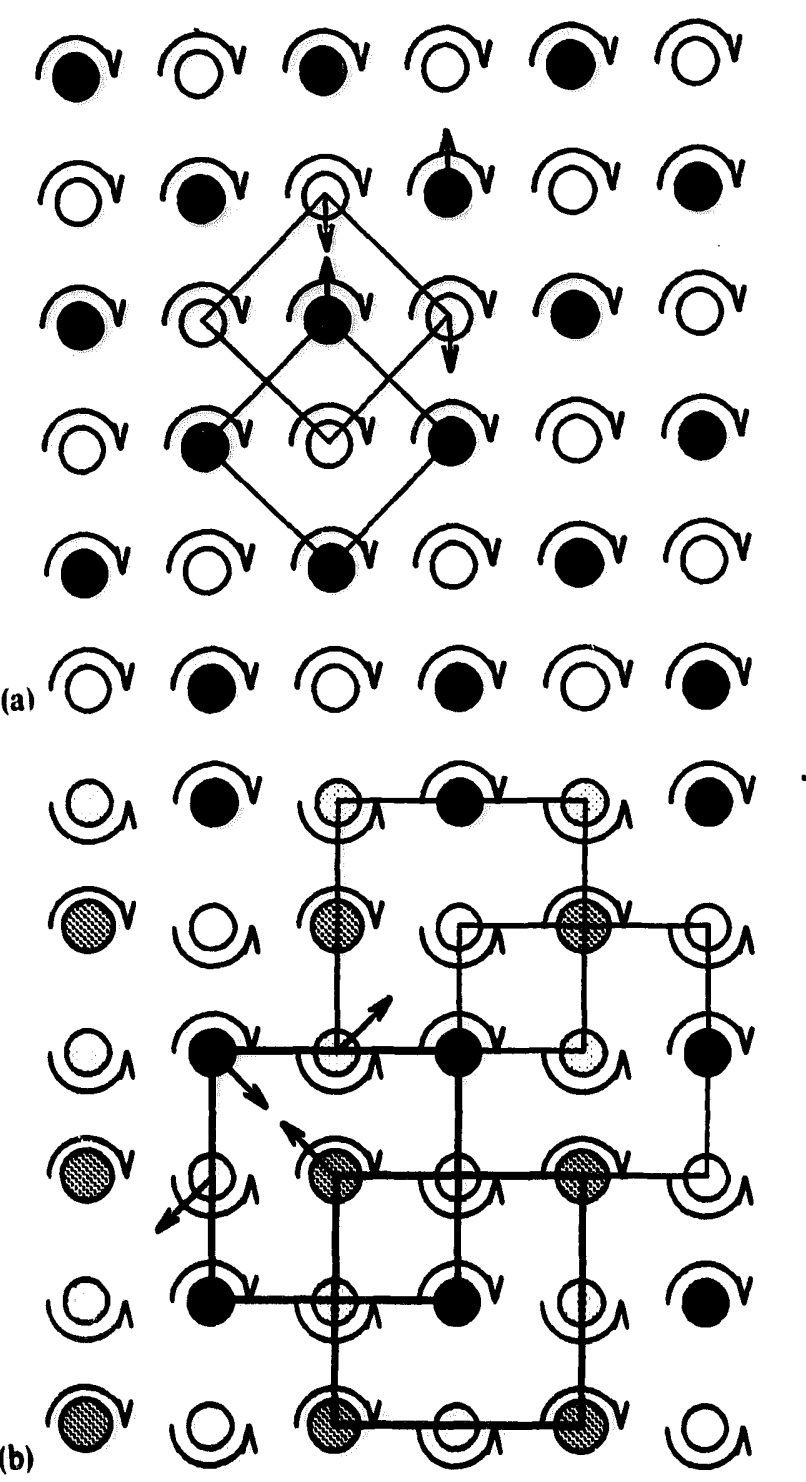

Fig. 6. Collective phase-locking modes. For each mode, the direction of rotation and the phase of the oscillators are represented using a surrounding arrow and grey pattern circle, respectively. Jets in phase are represented by same patterns and define sublattices whose mesh is drawn with either thin or thick lines, according to the direction of rotation. Square geometry: (a) diagonal mode, (b) gearing mode, (c) simultaneity mode. The arrows indicate the comparative instantaneous vector position of four neighbouring jets. Hexagonal geometry: (d) acoustic mode, (e) inscribed triangles mode, (f) asymmetrical $2 d$ hexagon mode, (g) $3 d$ hexagon mode. Triangular geometry: (h) acoustic mode, (i) parallel mode, (j) asymmetrical $2 d$ hexagon mode.

identified in the three geometries are represented in Fig. 6. For each mode, the direction of rotation and the phase of the oscillator are represented using a surrounding arrow and a grey pattern circle, respectively.

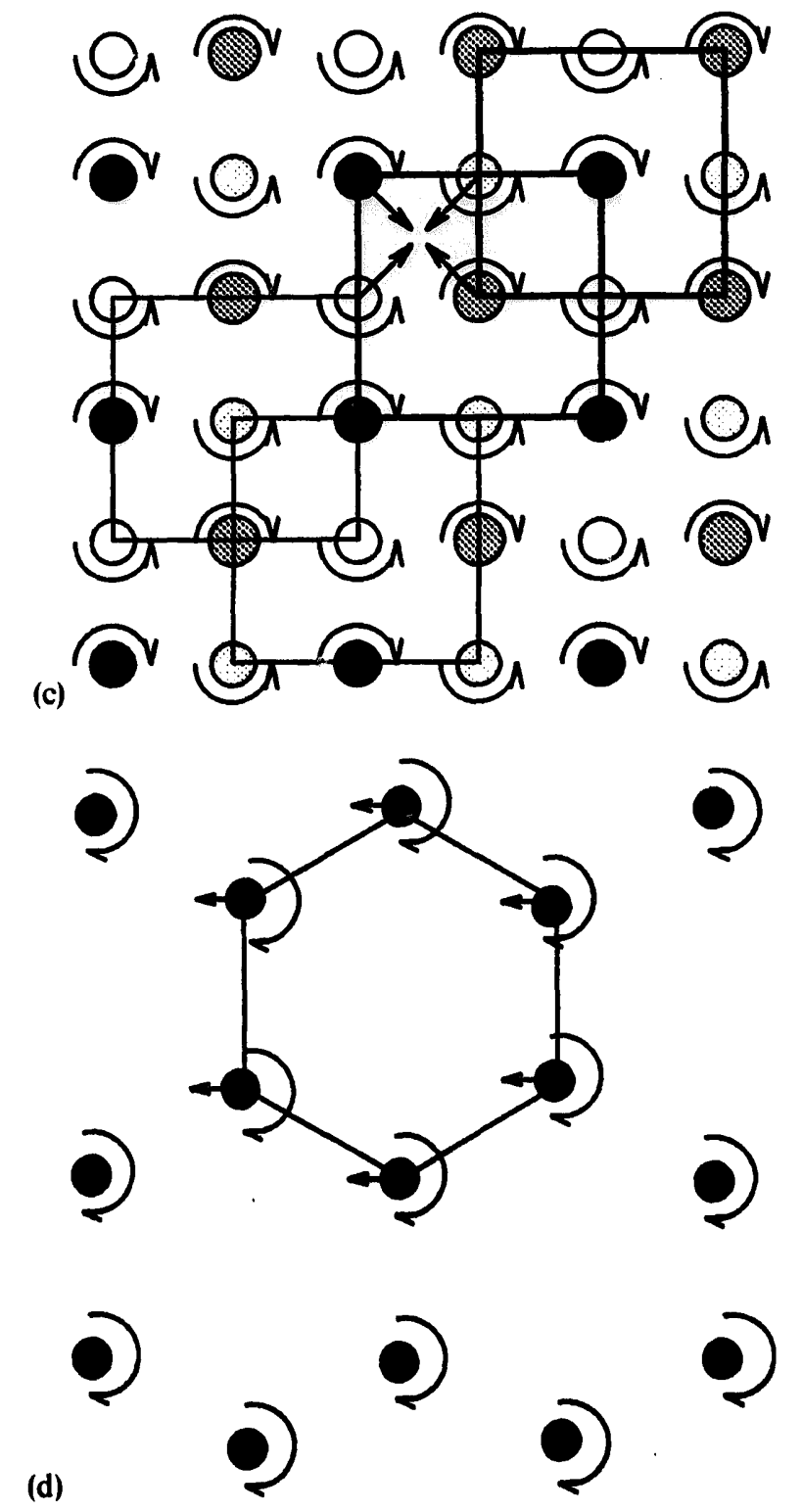

Fig. 6. Continued

Circles with the same grey pattern correspond to jets rotating in phase in the same direction.

In the square geometry, three modes have been identified: diagonal, gearing and simultaneity.

The diagonal mode is rather simple: all the jets rotate in the same direction (see Fig. 6(a) for the schematic representation, and Fig. 7 for the real system). The first neighbours of a given jet of reference are phase-shifted by $\pi$ with it, whereas its second neighbours are in phase with it. The jets in phase arrange thus in two sublattices phase-shifted of $\pi$, whose mesh is a square of size $\sqrt{2 d}$ (thin line squares of Fig. 6(a)). Arrows are used to represent 
18
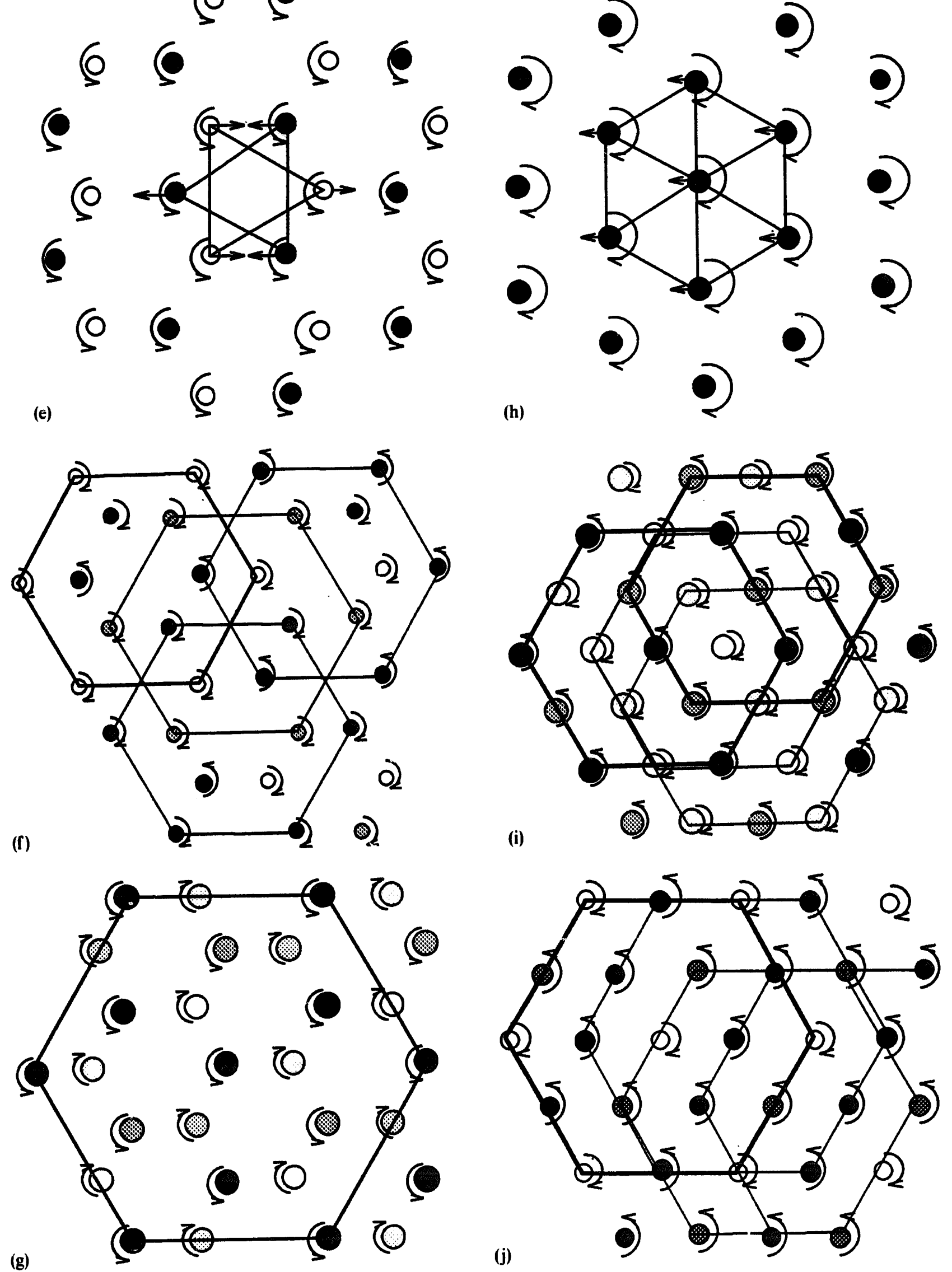

Fig. 6. Continued 
the instantaneaus position vector of the four nearest neighbours.

In the gearing (Fig. 6(b)) and simultaneity (Fig. 6(c)) modes, the jets arrange in two distinct subnetworks (one for each direction of rotation). Each subnetwork is subdivided into two $\pi$ phase-shifted sublattices where the jets rotate in phase in the same direction. The mesh of the sublattice is a square of size $2 d$ (thick and thin lines are used to distinguish the two subnetworks or the two directions of rotation). The first neighbours of a given reference jet rotate in the direction opposite to that of the reference, whereas the second neighbours rotate in the same direction but display a phase-shift of $\pi$ with it. The difference between the gearing and simultaneity modes is the comparative instantaneous position of the nearest neighbours: in the gearing mode (Fig. 6(b)), the arrows point alternatively at the centre of a four-jet unit, whereas in the simultaneity mode (Fig. 6(c)), they all point at the centre simultaneously, hence the respective names.

In the hexagonal geometry, four different modes have been identified: acoustic, inscribed triangles, asymmetrical $2 d$ hexagon and $3 d$ hexagon.

The acoustic mode (Fig. 6(d)) is the simplest one: all the jets rotate in phase in the same direction.

The inscribed triangles mode (Fig. 6(e)) is the transposition of the diagonal mode to the hexagonal geometry: the squares of size $\sqrt{2 d}$ are replaced by equilateral triangles of size $\sqrt{3 d}$.

The asymmetrical $2 d$ hexagon mode (Fig. 6(f)) is less symmetric than the previous ones. The jets arrange again in two distinct subnetworks (one for each direction of rotation), but one quarter of them rotate in the first direction whereas the other three rotate in the opposite direction. For the jets of references of the first type (blank circles of Fig. 6(f)), all the first neighbours rotate in the direction opposite to that of the reference, whereas for the jets of reference of the second type (black and grey circles), one-third of them rotate in the direction opposite to that of the reference and the other two-thirds rotate in the same direction. The jets in phase of the first type arrange in one sublattice of hexagons of size $2 d$. The jets in phase of the second type ar-

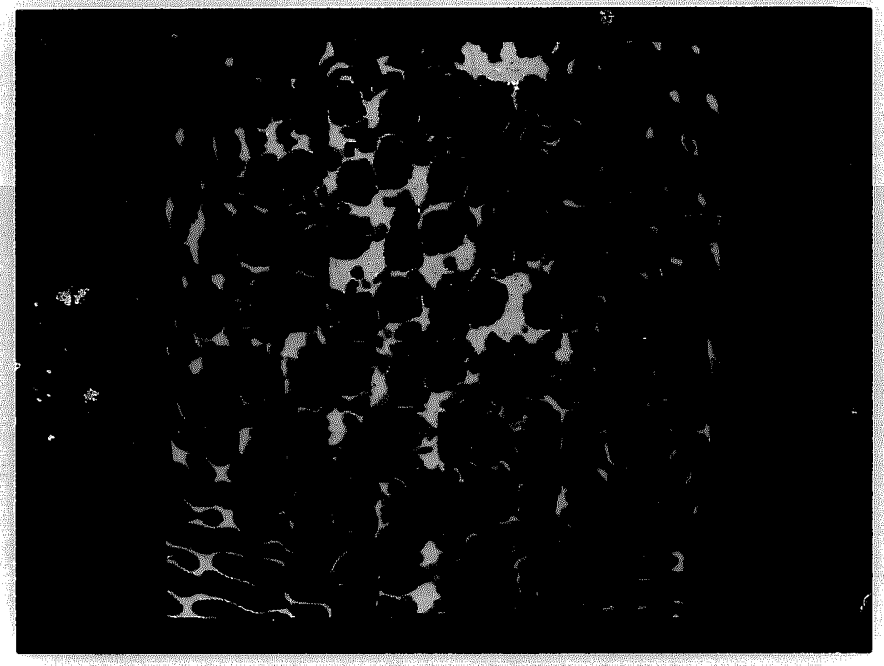

Fig. 7. Collective diagonal mode for a square geometry (seen from above).

range in three phase-shifted sublattices of hexagons of size $2 d$. The difference between all the previous modes is that two jets of reference (one of each type) are now needed to derive the spatial organization of the network using invariance by translation properties.

In the $3 d$ hexagon mode (Fig. 6(g)), the jets arrange in two distinct subnetworks too (one for each direction of rotation). Each subnetwork subdivides into three phase-shifted sublattices where the jets rotate in phase in the same direction. The mesh of the sublattice is a hexagon of size $3 d$. For both subnetworks, the first and third neighbours of a given reference jet rotate in a direction opposite to that of the reference, whereas the second neighbours rotate in the same direction but are phase-shifted with it. Note that in contrast with all the previous modes, the phase-shift of the second neighbours depends on the eigendirections of the network.

In the triangular geometry, three different modes have been identified: acoustic, asymmetrical $2 d$ hexagon and parallel.

The acoustic mode (Fig. 6(h)) is simply the transposition of the acoustic mode of the hexagonal geometry. The same remark stands for the asymmetrical $2 d$ hexagon mode (Fig. 6(i)) too.

The parallel mode (Fig. 6(j)) is specific to the triangular geometry. The jets arrange in two distinct subnetworks (one for each direction of rotation). Each subnetwork is subdivided into two $\pi$ phase-shifted 
sublattices where the jets rotate in phase in the same direction. The mesh of the sublattice is a hexagon of size $2 d$ (thick and thin lines are used to distinguish the two subnetworks, or the two directions of rotation). In contrast with all the previous modes, all the first neighbours of a given reference jet do not rotate in the same direction: one-third of them rotate in the same direction as the jet of reference, whereas the other two-third rotate in the opposite direction.

\subsubsection{Diagrams of stability}

For each set of plate parameters of Table 1, a stability diagram has been constructed, where the field of existence of the different collective modes is represented in a bidimensiona! flow rate $(F) /$ depth $(h)$ space. Two diagrams are represented for the square geometry (Fig. 8) and the hexagonal geometry (Fig. 9). The diagrams are built up using the following procedure: the flow rate is decreased by. regular steps, and for each flow rate value, the plate is lifted by $2 \mathrm{~mm}$ steps, from a depth of $3 \mathrm{~cm}$ to the surface (the transition for a single jet is not observed deeper). For each diagram, a symbol representing the state of the system is reported for each couple of discrete $\left(F_{i}, h_{j}\right)$ values of the parameters $F$ and $h$. The filled-square symbols correspond to low flow rate values (below the threshold): no individual or collective instability is observed. The filleddiamond symbols correspond to high flow rate values: each water jet is in a fountain regime and no collective mode is observed. In-between, each symbol zone represents a particular collective mode among those described above. Plus symbols represent regimes intermediate between two well determined phase-locking modes. Cross symbols represent regimes with intermittent coupling of the bumps: such regimes are observed just above the threshold. In Fig. 8(a), the dashed curve represents the variation of the flow rate threshold versus the depth of the plate: note that the threshold increases roughly linearly with the depth.

For the square geometry (Figs. 8(a) and (b)), the diagonal, gearing and simultaneity modes are represented by circle, square and triangle-right symbols, respectively. Two other modes have been observed marginally at rather shallow depths: triangle-up symbols correspond to an optical mode between the lines
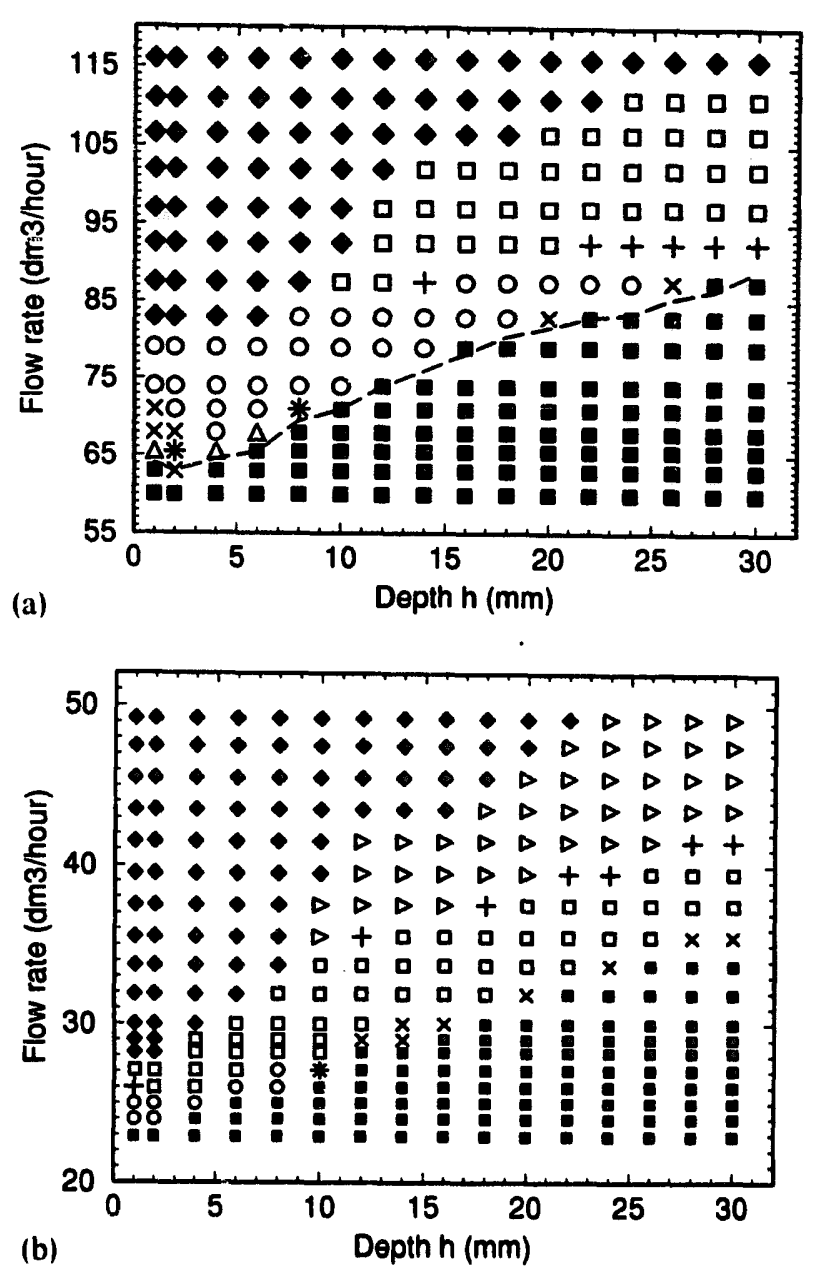

Fig. 8. Collective modes' stability diagrams - Square geometry: (a) $N=57, d=12 \mathrm{~mm}, \phi=1.0 \mathrm{~mm}$; (b) $N=57 ; d=6 \mathrm{~mm}$; $\phi=0.5 \mathrm{~mm}$. no collective dynamics; : fountain regime; $x$ : nonpermanent regime; + : unidentified regime; $\circ$ : diagonal mode; $\square:$ gearing mode; $\triangleright:$ simultaneity mode; $\Delta:$ optical mode; *: shear mode.

(or the columns) of the square network, whereas star symbols correspond to a shear mode between the lines (or the columns). Note that in these two modes, the jets are in the sinuous instability mode and not in the helical mode as for all the previous collective modes. The plate parameters for the diagram of Fig. 8(a) are $N=57, d=12 \mathrm{~mm}$ and $\phi=1.0 \mathrm{~mm}$, whereas those for Fig. 8(b) are $N=57, d=6 \mathrm{~mm}$ and $\phi=0.5 \mathrm{~mm}$ (Table 1). Note that the $d / \phi$ ratio $(d / \phi=12)$ is kept constant for both diagrams.

First, a comparison between the two diagrams shows that the field of existence of the different collective modes is shifted. Moreover, the simultaneity mode is observed in Fig. 8(b), but not in Fig. 8(a). 

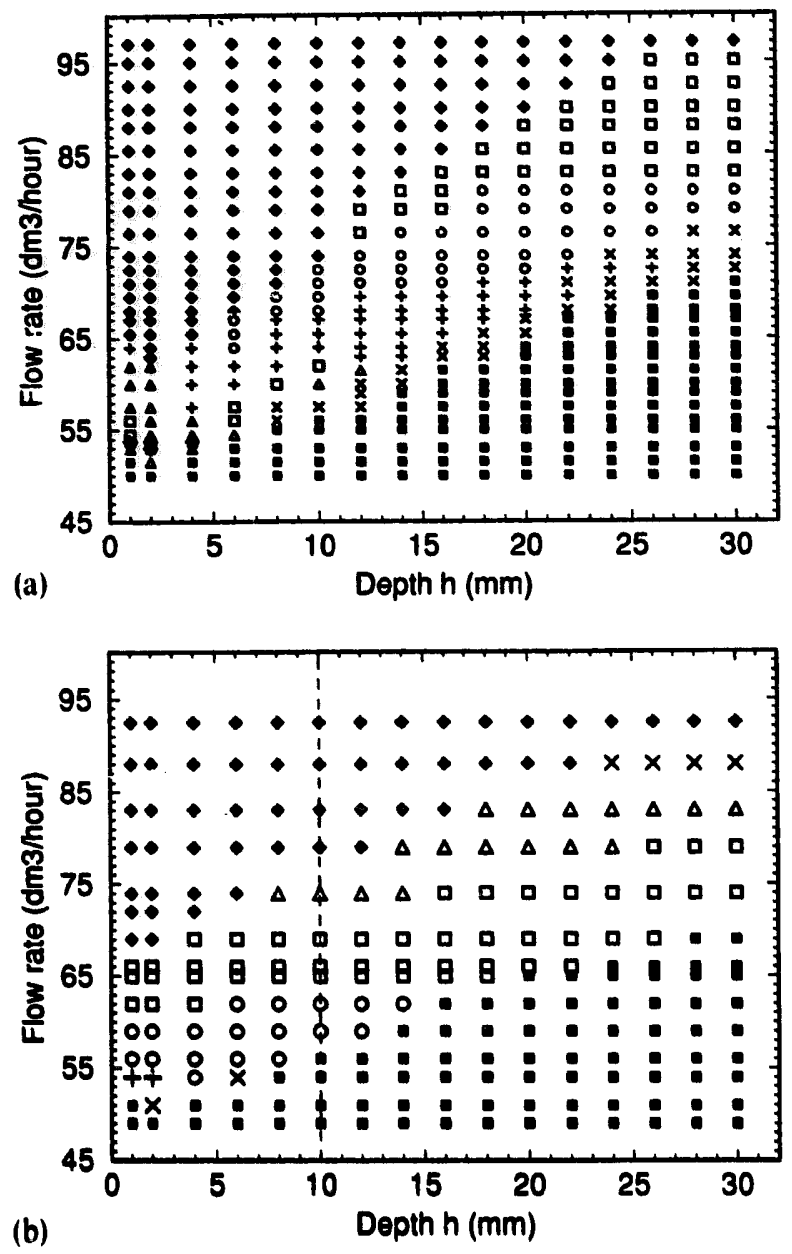

Fig. 9. Collective modes' stability diagrams - Hexagonal geometry: (a) $N=46, d=12 \mathrm{~mm}, \phi=1.0 \mathrm{~mm}$; (b) $N=46$, $d=8 \mathrm{~mm}, \phi=1.0 \mathrm{~mm} . \square$ : no collective dynamics; $\$$ : fountain regime; $x$ : nonpermanent regime; $+:$ unidentified regime; o: acoustic mode; $\square: 3 d$ hexagon mode; $\Delta:$ inscribed triangles mode; *: asymmetrical $2 d$ hexagon mode. The same scaling is applied for both panels (a) and (b).

Second, the same modes can be observed for flow rate values far different: the diagonal and gearing modes are observed in both diagrams 8(a) and (b), corresponding to 1.0 and $0.5 \mathrm{~mm}$ diameter jets, respertively. Third, for each diagram, transitions are observed from one mode to another when the flow rate $F$ (alone) or the depth $h$ (alone) is modified: note for instance the transition from the gearing to the simultaneity mode when the flow rate increases, for a depth of $10 \mathrm{~mm}$, or when the depth decreases for a flow rate of $37.5 \mathrm{dm}^{3} / \mathrm{h}$ (Fig. 8(b)).

For the hexagonal geometry (Figs. 9(a) and (b)), the acoustic, inscribed triangles, $3 d$ hexagon and asym- metrical $2 d$ hexagon modes are represented by circle, triangle-up, square and star symbols respectively. In this geometry, no collective mode corresponding to the sinuous instability mode of the jets has been observed. The plate parameters for the diagram of Fig. 9(a) are $N=46, d=12 \mathrm{~mm}$ and $\phi=1.0 \mathrm{~mm}$, whereas those for Fig. 9(b) are $N=46, d=8 \mathrm{~mm}$ and $\phi=1.0 \mathrm{~mm}$. This time, only the distance $d$ between nearest jets has been changed (from 12 to $8 \mathrm{~mm}$ ). The significance of the vertical dashed line drawn for a depth of $10 \mathrm{~mm}$ will be examined in the next section.

A comparison between the two diagrams shows, again, that the field of existence of the different collective modes is shifted. Transitions from one mode to another are observed, again, when the flow rate $F$ (alone) or the depth $h$ (alone) is changed: note for instance the transition from the acoustic to the $3 d$ hexagon, then from the $3 d$ hexagon to the inscribed triangles mode when the flow rate is increased for a depth of $10 \mathrm{~mm}$ (Fig. 9(b)).

\subsection{Mechanism of coupling between the jets}

Two major questions arise concerning the coupling between the jets: (i) By what mechanism do the oscillating jets couple to each other? (ii) What governs the transitions that are observed between the different collective modes?

In the bidimensional system of co-flowing air-jets of Villermaux and Hopfinger [26], the existence of a fluid recirculation cavity due to the confinement of the jets by their nearest neighbours is crucial to the phenomenon of individual and collective oscillation. In the present experiment, the 3D recirculation of the jets does not seem to be of crucial importance. Indeed, a given collective mode can be observed with either a system of tubes (see Section 2) or perforated plates, and for depths of either 1 or $3 \mathrm{~cm}$, providing quite different recirculating patterns for the jets. On the other hand, no coupling between the jets has ever been observed in the absence of surface waves radiating from the oscillating bumps. As pointed out above for two-jet systems, the coupling between the jets seems to be stronger when the jets are set deep below the surface, generar ng stronger surface ripples. 
Surface waves could thus convey the interaction between the jets, providing long distance rather than diffusive coupling of the oscillators.

Concerning the transitions observed between the different modes in the stability diagrams, a first intuitive interpretation is that the bifurcations result from the coritinous increase (or decrease) of a pertinent coupling parameter. Since surface waves provide the most plausible mechanism of coupling, the pertinent coupling parameter should include some of the characteristics of the waves.

The experiment is governed by three parameters: the flow rate $F$, the depth $h$ and the distance $d$ between nearest neighbours (for a given geometry). Using the different stability diagrams obtained for the sets of plate parameters of Table 1 , the respective role of the three parameters is highlighted using three different transformations where one of the three parameters is changed while the other two are left constant. We consider thus transformation $\alpha: h$ and $d$ constant, $F$ increases, transformation $\beta: F$ and $d$ constant, $h$ decreases and transformation $\gamma: F$ and $h$ constant, $d$ decreases. For each geometry, the same sequence of bifurcations from one mode to another is observed for the three transformations:

square geometry - diagonal $\rightarrow$ gearing $\rightarrow$ simultaneity mode; hexagonal geometry - plus symbol $\rightarrow$ acoustic $\rightarrow$ 3d hexagon $\rightarrow$ inscribed triangles mode; triangular geometry - parallel $\rightarrow$ acoustic mode.

Note that the validity of the above sequences can be partially checked using the stability diagrams of Figs. 8 (square geometry) and 9 (hexagonal geometry). The term "partially" stems from the fact that a complete sequence is never observed along a single horizontal or vertical profile of a stability diagram (at best, two transitions can be observed along the same profile: vertical dashed line of Fig. 9(b)). To derive the above sequences, a complete set of stability diagrams is thus required.

A surprising feature is observed, which is not represented here: in many cases, the sequence of bifurcations loops, so that increasing (or decreasing) again the single parameter of the transformation induces the first mode of the series to replace the last one. This is observed for transformations $(\alpha)$ and $(\beta)$ in the square geometry, for transformation $(\alpha)$ in the hexagonal geometry, and for transformations $(\alpha),(\beta)$, and $(\gamma)$ in the triangular geometry. Finally, collective modes with very reduced existence fields in the diagrams (optical and shear modes in the square geometry, and asymmetrical $2 d$ hexagon modes in the hexagonal and triangular geometries) have not been included in the present analysis.

Different coupling scenarios are now considered, whose validity is examined with regard to the above sequence of modes. First, the role of the flow rate $F$ and of the jet velocity $u_{0}$ at the nozzle exit is examined briefly: as pointed out above in the comparison of diagrams 8(a) and (b), the same collective mode can be observed for very different flow rate values: this shows that the flow rate is not a relevant quantity for the coupling between the jets. The mean velocity $u_{0}$ at the jet exit is neither a relevant quantity: transitions are observed between modes when $d$ is decreased at constant $F$ and $\phi$ values (and thus at constant $u_{0}=$ cte $^{*} F / \phi^{2}$ ). Finally, the marked difference between diagrams 8(a) and (b) shows that $d / \phi$ is not a relevant quantity either.

The next scenario was to consider the height of surface bumps as a possible quantity relevant to coupling. First, the oscillatory mode for a single bump starts when the height of the bump reaches a threshold value (Fig. 2). Second, the same height can indeed be obtained for flow rate values far different if the diameter of the jet is changed, like in Figs. 8(a) and (b). Third, the frequency of the oscillatory mode and the height of the bump are correlated (Section 3.2). In the case of a single jet, we have verified that when the flow rate (alone) increases, or when the depth (alone) decreases, the height of the bump increases. The sequence of modes observed for both transformations $\alpha$ and $\beta$ could then be interpreted as a consequence of the continuous increase of bump height. However, this hypothesis breaks down when transformation $\gamma$ is considered: when the distance between the jets is reduced, at constant $F$ and $h$, the height of the bumps is left unchanged, whereas transitions between collective modes are still observed.

Let us now consider that the coupling is not governed by the height of the bump itself but by the 


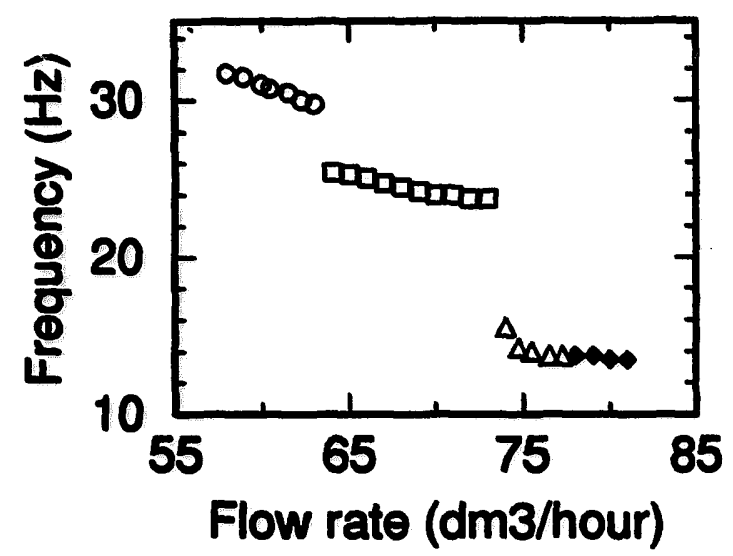

Fig. 10. The frequency of the collective modes is measured across the vertical dashed line profile of Fig. 9(b), for increasing values of the flow rate above the threshold. The symbols are the same as in Fig. 9(b). Note the marked jump of frequency each time the system switches to a new mode.

amplitude of the surface ripples that are generated at a distance $d$ from the radiating bump (see Section 3). Note that this is equivalent to considering an adimensional coupling parameter constructed as the ratio of the bump height to the distance $d$ of the first neighbours. This explanation is still coherent with the sequence of modes observed for both transformations $\alpha$ and $\beta$ : if the height of the bump increases, the amplitude of the surface ripples increases in the same way. It is also coherent with the sequence observed for transformation $\gamma$ : the conservation of energy implies a decrease of the amplitude of the ripples when the radial distance from the point source increases. Thus, if the jets are brought closer, the amplitude of the ripples at the distance of the first neighbours increases, in agreement with the sequence of bifurcations. However, this scenario does not account for the looping of the sequence of modes.

Anyway, surface waves remain the most plausible means of coupling. One should thus consider the thorough interaction between an oscillating jet and the surface waves that propagate from all the other jets of the network, in particular resonance effects such as the tuning between the wavelength of the collective modes and the discrete wavelengths of the network. This tuning effect could well explain the looping phenomenon observed for the sequence of modes, and would provide a valuable insight into the mechanism of transition from one mode to another. In Fig. 10, the frequency of the collective modes has been measured along the vertical dashed-line profile of Fig. 9(b), using a photodiode and a spectrum analyser (same set-up as in Section 3). The variations of the frequency are plotted for increasing flow rate values above the threshold, for a depth of $10 \mathrm{~mm}$. The symbols are the same as those used in Fig. 9(b). When the system remains in the same phase-locking mode, the frequency decreases smoothly with increasing flow rate. However, each time the system switches to another mode (from the acoustic to the $3 d$ hexagon mode, then from the $3 d$ hexagon to the inscribed triangles mode), a sharp drop of frequency occurs. Now decreasing the flow rate in a reverse path, a slight hysteresis phenomenon is observed, which is not represented in Fig. 10. In contrast with the case of a single jet (Section 3), the sharpness of the collective mode does not decrease when the flow rate above the onset of the mode increases. The peak of frequency in the spectrum remains narrow instead. In our opinion, this results from the possibility of bifurcating from one collective mode to another.

At this stage, we propose the bifurcation from one mode to another to be governed by a tuning criterion between the wavelength of the oscillatory mode of the jets and the discrete wavelengths associated with the periodic bidimensional pattern of the network. The mechanism might be as follows: the frequency, and hence the wavelength of the oscillatory mode of the jets are governed by external variations of the parameters $F$ and $h$ and by the surface waves. The system adjusts until the tuning of the present collective mode drops below that of a new collective mode, with some hysteresis however. Then the system bifurcates to the new mode.

\subsection{Considerations of symmetry}

General conclusions can be drawn from the description of the collective modes (Section 4.2.1):

- the jets always oscillate at the same frequency and with the same amplitude,

- the sublattices defined by the jets in phase are regular polygons whose symmetry is that of the perforated plate, 
- the mesh of all different sublattices is the same,

- the spatial arrangements display no modulation, and thus look similar to those encountered in crystals (for example, the gearing mode of Fig. 3(a) is the analogous of a $\mathrm{NaCl}$ crystallographic structure).

All this is relevant to a strong coupling between the jets. However, the coupling is not strong enough to perturb the limit cycles of the oscillators [4]. Although a mechanism has been proposed in the previous section to explain the transitions between the collective modes, the criteria of selection of the particular topology of the collective modes (see Section 4.2.!) are still unclear. For example, the diagonal (square geometry) and inscribed triangles (hexagonal geometry) modes have no equivalent in the triangular geometry, and the gearing and simultaneity modes have no equivalent in the hexagonal and triangular geometries.

Using considerations of symmetry, it is indeed possible to explain some of these features. To investigate the symmetries of the system of coupled water jets, two different approaches are considered: the reference jet approach and the group theory approach. The first one is rather specific to the present experiment and derives from the experimental procedure adopted to observe the collective modes. The second one is more generic and examines the spatio-temporal symmetry groups of the collective modes.

\subsubsection{Reference jet approach}

This approach derives naturally from the observations: it stems from the fact that the collective modes display invariance by translation properties. Indeed, except for the asymmetrical $2 d$ hexagon mode, the spatial arrangement of the network can be reconsiructed using one reference jet only, a basic pattern for its nearest neighbours, and invariance by translation properties. Now/ using this invariance by translation property as a basic postulate, a systematic investigation of all the possible modes is undertaken. With this aim, the foliowing procedure has been adopted: a given jet is taken as a reference, and a systematic investigation of all the possible patterns defined by the comparative state of its nearest neighbours is carried out. The comparative state of a

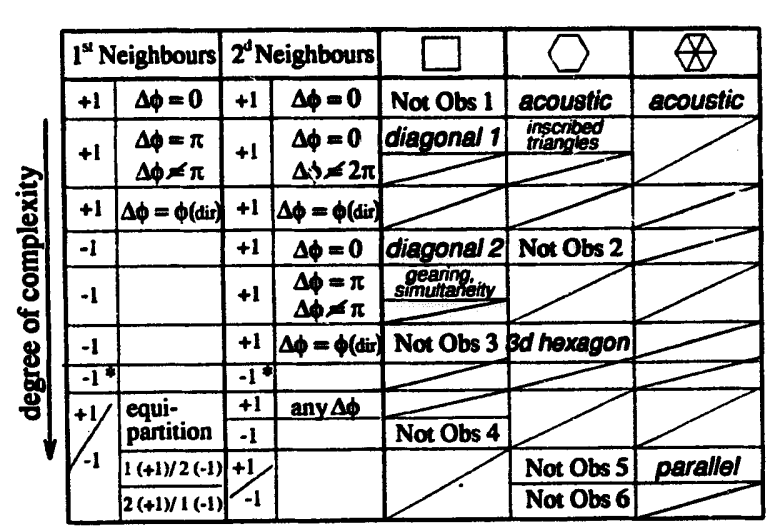

Fig. 11. Schematic representation of the systematic scheme used to investigate the symmetries of the system. A given jet is taken as a reference, and the comparative state of its first and second neighbours is explored (comparative direction of rotation: " -1 " if opposite, " +1 " if same as reference, and for the latter comparative phase shift with the reference jet $\Delta \phi$ ). The corresponding spatial mode is reported for the three geometries. The degree of complexity increases from top to bottom. When the spatial mode violates the basic hypothesis of invariance by translation, an oblique line is drawn in the corresponding box.

neigbouring jet is, again, characterized by the comparative direction of rotation of its helical mode and, if same direction, by its phase-shift with the reference jet. The procedure is thus the same as that applied for the observations (Section 4.2.1.), but it is extended here to a systematic and comprehensive investigation.

The systematic investigation is summarized in detail in Fig. 11. The two columns "first neighbours" and "second neighbours" at the left describe the comparative state of the first and second neighbours of the jet of reference. The " +1 " ("-1") signify that the neighbouring jets rotate in the same (opposite) direction as that of the reference jet. When in the same direction, their phase-shift with the reference jet is indicated by the $\Delta \phi$ value. When the phase-shift is not isotropic but takes different values along the different eigendirections of the network, a $\Delta \phi=\Delta \phi$ (dir) designation is used. In the three columns at the right, the corresponding spatial arrangement of the jets is reported for the three network geometries. Three cases can be distinguished: (i) when the corresponding spatial arrangment violates the basic hypothesis of invariance by translation, an oblique line is drawn across the box, (ii) when the spatial arrangement has been observed experimentally and is one of the collective 
modes described in Section 4.2.1, the specific name given in Section 4.2.1 is reported, in italics (iii) when the spatial mode has not been observed, a "Not Obs" designation is used.

The degree of compiexity of the pattern defined by the first and second neighbours increases from the top to the bottom of Fig. 11. To begin with, the simplest pattern is considered: all the first neighbours rotate in the same direction as that of the reference jet ("+ 1 ") and are in phase with it. The following step towards more complexity is to consider that all the first neighbours rotate in the same direction as that of the reference jet, but present a same phase-shift $\Delta \phi$ with it. The step further is then to consider that the phase-shift is not the same for all the first neighbours, but takes different values according to the different eigendirec- tions of the network (two in the square geometry, three in both hexagonal and triangular geometries): $\Delta \phi=\Delta \phi_{i}$. As a consequence of the invariance by translation property, the second neighbours rotate in the same direction as that of the reference ("+1") but can be phase-shifted with it. Actually, the comparative state of the third, fourth and even farther neighbours could also be considered. In the present investigation, only first and second neighbours have been retained, although the analysis has been extended to the third neighbours in some cases. This was motivated by the small size of the experimental system of coupled oscillators ( $N$ ranges from 46 to 89 in Table 1).

In a next step, the first neighbours rotate all in the direction opposite to that of the reference ("-1") whereas the second neighbours still rotate in the same direction as that of the reference ("+l"). In analogy with the previous scheme, different cases can be considered according to the comparative phase-shift of the second neighbours with the reference jet. The cases of zero, non-zero and direction dependent phaseshifts are thus considered successively for the second neighbours. A logical continuation of the investigation scheme is then to consider that both first and second neighbours rotate in the direction opposite to that of the reference jet ("-1", "-1").

Finally, in a last step, the case where the first neighbours of the reference jet do not rotate all in the same direction is examined. A further subdivision is made depending on whether the two directions of rotation are partitioned equally among the first neighbours or not.

Using Fig. 11, let us now compare our predictions to the observations:

First, on the 10 collective modes of Section 4.2.1 (helical instability mode of the jet) eight are predicted using the systematic scheme of Fig. 11. The complex spatial arrangements presented in Fig. 6 are retrieved exactly, which post-validates the observations. The two collective modes which do not appear in Fig. 11 are the asymmetrical $2 d$ hexagon modes, observed for the hexagonal and triangular geometries. Actually, these can be recovered using a slightly different investigation scheme. Indeed, if two different types of reference jet are now considered, with a distinct pattern for the first and second neighbours, new collective modes can be predicted, among them the asymmetrical $2 d$ hexagon mode, that still satisfy some invariance by translation properties (although in a weaker sense). In the asymmetrical $2 d$ hexagon mode, one of the two reference jets has the (" $-1^{* ”, ~ " ~}-1^{* ”)}$ pattern of Fig. 11 for the columns "first neighbours" and "second neighbours", whereas the other one has the $2(+1) / 1(-1)$ pattern of Fig. 11 for the column "first neighbours". This can be verified in Fig. 6(j) for instance, where the blank circle symbols have the (" $-1^{* \prime,}, "-1^{* * \prime)}$ pattern, whereas all the grey circle symbols have the $2(+1) / 1(-1)$ pattern.

Second, using the systematic investigation scheme of Fig. 11, it is possible to explain some of the discrepancies observed between the square, hexagonal and triangular geometries. As pointed out above in the text, the diagonal (square geometry) and inscribed triangles (hexagonal geometry) modes have no equivalent in the triangular geometry, whereas the gearing and simultaneity modes (square geometry) have no equivalent in the hexagonal and triangular geometries.

In both the diagonal and inscribed triangles modes, all the first neighbours present the same phase-shift with the reference jet, $\Delta \phi=\pi$, Fig. 11. The invariance by translation satisfied by these modes implies that the same first neighbours must also display a phase-shift of $\pi$ with their own first neighbours. This requires, among other things, the first neighbours not 


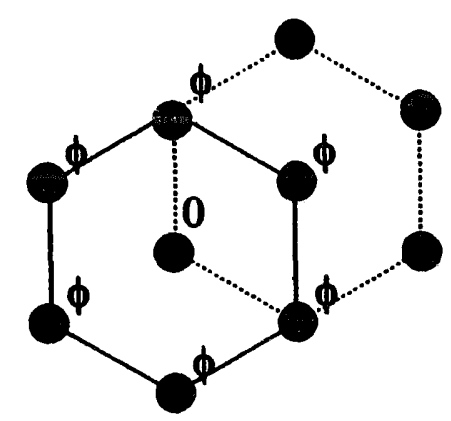

Fig. 12. Illustration of noninvariance properties for a triangular geometry.

to be first neighbours among them. If this is verified in the square and hexagonal geometries (see Figs. 6(a) and (e) for instance), this is not the case in the triangular geometry. This is illustrated in Fig. 12: a given jet is taken as a reference, and its first six neighbours are phase-shifted by $\phi$ with it (full line hexagon). If one of the first neighbours of the reference jet is now taken as the new reference, two of its own first six neighbours (dashed line hexagon) are then in phase with it. This is the reason why a mode equivalent to the diagonal or inscribed triangles modes is not observed in this geometry.

In the gearing or simultaneity modes (square geometry), the first neighbours of a given reference jet rotate all in the direction opposite to that of the reference. Again, for the same reason as that mentioned above, this state of things is not possible in the triangular geometry. For the hexagonal geometry however, no problem occurs with the first neighbours since each jet has three first neighbours which are only second neighbours between them. In the gearing mode (Fig. 6(b)), the second neighbours of a given reference jet rotate in the same direction as that of the reference but display all the same phase-shift $(\pi)$ with it. For a hexagonal network, the second neighbours of a given reference jet define a triangular sublattice: using again the same argument as before (for the second neighbours this time), a mode equivalent to the gearing or simultaneity modes in the square geometry cannot be observed in the hexagonal geometry.

The third point is that some spatial collective modes are predicted that are not observed. They correspond to the "Not Obs" designation in Fig. 11. In most cases, modes with the same symmetry are observed in other geometries. This is the case for the acoustic and $3 d$ hexagon modes in the square geometry, and for the diagonal 2 and parallel modes in the hexagonal geometry.

Several reasons can be invoked:

- First, the range of plate parameters has not been fully explored: for instance, the diagonal 2 mode has been observed with a 13-jet-square network, but never with the 57-jet-square network. Networks with so small a number of jets have been tested in the square geometry only, explaining perhaps the "Not Obs" mode No. 2 in the hexagonal geometry.

- Second, very complex (poorly symmetric) spatial organizations are unlikely to be stable given the small size of the system ( $N$ ranges from 46 to 89 in Table 1). For instance, in the "Not Obs" mode No. 4, jets in-phase arrange in two subnetworks of four phase-shifted sublattices whose mesh is a square of size $2 \sqrt{2 d}$. In the same way, in the "Not Obs" No. 6 , jets in-phase arrange in two subnetworks of four phase-shifted sublattices whose mesh is a hexagon of size $2 \sqrt{3 d}$. Similarly, collective modes of intermediate complexity may not be observed because simpler modes (more symmetric) already exist. This is the case of the "Not Obs" mode No. 3 in the square geometry, where the diagonal, gearing and simultaneity modes are observed, and of the "Not Obs" mode No. 5 in the hexagonal geometry, where the acoustic, inscribed triangles and $3 d$ hexagon modes are already observed. Note that, in constrast, no collective mode of intermediate complexity is predicted in the triangular geometry, and that a complex parallel mode is observed.

- Third, and perhaps the most important, the present symmetry considerations do not take into account the comparative stability of the different modes. However, we see no obvious reason why the acoustic mode ("Not Obs" mode No. 1) should not be observed in the square geometry.

\subsubsection{Group theory approach}

The second approach is more general, and has been widely used in crystallography or in the description of 
the symmetries of systems of coupled oscillators [37]. A group theory approach has been recently proposed to analyse the symmetries of animal gaits $[14,15]$. Since the reference jet approach leads to conclusive results already, our purpose here is not to present a comprehensive treatment of the symmetries of the system using group-theoretics, but to introduce the key points necessary to the specific treatment of the system of coupled water jets. The analysis will thus be limited to the case of a square geometry.

Although the treatment is derived from that of a system of four coupled oscillators, such as in the problem of the quadruped locomotion [14], some features are specific to the present system. First, the size of the system $(N \approx 50)$ is too large for a comprehensive classification of the groups of symmetry of the global system to be carried out. Second, for each oscillator, two directions of rotations are possible, so that both the direction of rotation and the phase of the oscillator are needed to characterize its state.

To investigate the spatio-temporal symmetries of the present system, cyclic permutations of the oscillators, temporal phase-shifts, translations along the eigendirections of the bidimensional lattice and symmetries respective to a plane perpendicular to the plane of the network have to be composed. The former two are used to describe the symmetries of a system of four oscillators in the quadruped locomotion problem [14]. The latter two are specific to the present system. Indeed, since the oscillators are arranged periodically in a bidimensional square lattice (Figs. 6(a)-(c)), a logical issue is to combine the spatio-temporal symmetries of a basic four-jet system (Fig. 13(a)) with invariance by translation properties. Moreover, to describe the spatio-temporal symmetries of the four-jet system of Fig. 13(a), symmetries across a plane perpendicular to the plane of the figure are required to relate jets which rotate in opposite directions, like in the gearing and simultaneity modes.

The state of a given oscillator $i$ can be characterized by its direction of rotation $\left(\varepsilon_{i}= \pm 1\right)$ and by its phase $\left(\theta_{i}=\theta_{i}(t)\right)$. The latter is represented by position vectors in Fig. 13.

Thus consider the four-jet system of Fig. 13(a), where the os:illators are numerated from 1 to 4 . Let $\sigma$

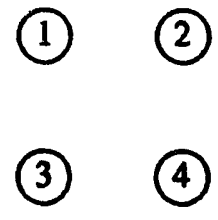

(a)

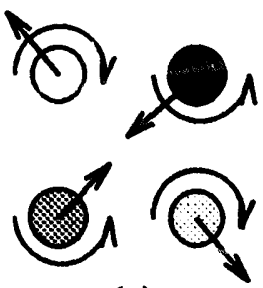

(c)

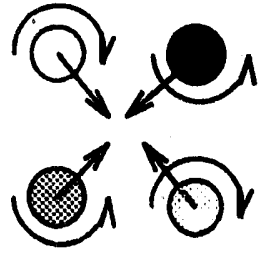

(e)

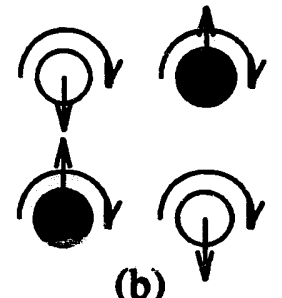

(b)

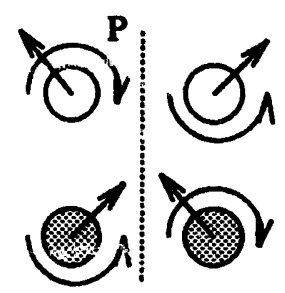

(d)

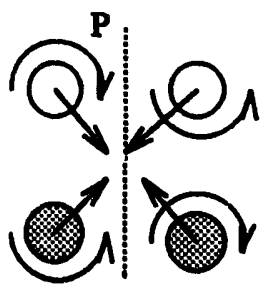

(f)
Fig. 13. Description of the symmetries of the collective modes using group theoretics: (a) basic system with four oscillators, (b) diagonal mode pattern, (c) gearing mode pattern, (d) left: oscillators 1 and 3 in the gearing mode pattern; right: state of oscillators 1 and 3 obtained after symmetry with respect to the plane $\boldsymbol{P}$, (e) simultaneity mode pattern, (f) left: oscillators 1 and 3 in the simultaneity mode pattern; right: state of oscillators 1 and 3 obtained after symmetry with respect to the plane $\boldsymbol{P}$.

be a permutation which sends oscillator $i$ to oscillator $i^{\prime} . \sigma$ can be decomposed into three elementary (cyclic) permutations: $\sigma_{1}=(12)(34)$ which interchanges oscillators 1 and 2, and 3 and 4 (left-right reflection), $\sigma_{2}=(13)(24)$ which interchanges oscillators 1 and 3, and 2 and 4 (front-back reflection), and $\sigma_{3}=\sigma_{1} \sigma_{2}=$ (14)(23), their composite, which is an interchange across diagonals. I is the trivial permutation which sends oscillators $i$ to $i$. Let $S$ be the symmetry respective to the plane $P$, which is the median plane of the line segment connecting oscillator $i$ to oscillator $i^{\prime}$. $\boldsymbol{P}$ is perpendicular to the plane of the figure, and has been represented in Figs. 13(d) and (f) permutation $\sigma_{1}$. 
Note that $S^{2}=I$, the identity transformation. More generally, $S^{p}=I$ if $p$ is even, and $S^{p}=S$ if $p$ is odd. Let $T_{n, m}$ be the translation which sends oscillator $i$ with cartesian coordinates $\left(x_{i}, y_{i}\right)$ to oscillator $j$ with cartesian coordinates $\left(x_{j}=x_{i}+n d I ; y_{j}=y_{i}+m d J\right)$, where $d$ is the distance between nearest jets, and $I$ and $J$ are unit vectors in the $x$ and $y$ directions respectively. Finally, Let $\tau_{\phi}$ be the translation which shifts time from $t$ to $t-\phi$.

$\sigma S^{p} T_{n, m} \tau_{\phi}$ is a spatio-temporal symmetry of the system if

$\left(\varepsilon_{i}, \theta_{i}(t)\right)=T_{n, m} S^{p}\left(\varepsilon_{i^{\prime}}, \theta_{i^{\prime}}(t-\phi)\right)$.

Using compositions of $\sigma, S^{p}, T_{n, m}$ and $\tau_{\phi}$, a comprehensive list of all the symmetry groups of the system of four jets could be carried out [14]. However, our purpose here is only to illustrate the method in the present case in a few examples.

The spatial arrangements corresponding to the $d i$ agonal, gearing and simultaneity collective modes are represented in Figs. 13(b), (c) and (e), respectively, for a four-jet system. Their symmetries can be derived easily using the symmetry groups.

The symmetries of the diagonal mode (Fig. 13(b)) are thus:

- pure permutations: the trivial permutation $I$ and the composition $\alpha \beta$,

- spatio-temporal compositions: $\tau_{\pi} \sigma_{1}, \tau_{\pi} \sigma_{2}$,

- pure plane symmetries: since all the jets rotate in the same direction, $I$ is the only plane symmetry of the mode,

- pure translations: $T_{n, m}$ (with $n$ and $m$ multiple of 2d).

Note that the symmetries composed with translations $T_{1,0}$ and $T_{0,1}$ are equivalent to the symmetries composed with the cyclic permutations $\sigma_{1}$ and $\sigma_{2}$ :

$\tau_{\pi} \sigma_{1}=\tau_{\pi} T_{1,0} ; \quad \tau_{\pi} \sigma_{2}=\tau_{\pi} T_{0,1}$

and have thus not been considered.

The gearing and simultaneity modes (Figs. 13(c) and (e)) share the same symmetries:

- pure permutations: the trivial permutation $I$,

- spatio-temporal compositions: $\tau_{\pi} \sigma_{1} \sigma_{2}$,

- pure translations: $T_{n, m}$ (with $n$ and $m$ multiple of $2 d$ ).
Table 2

\begin{tabular}{lll}
\hline & Gearing mode & Simultaneity mode \\
\hline Pure plane symmetries & $I$ & $S, S^{\prime}, I$ \\
Compositions & $\tau_{\pi} S \sigma_{1}, \tau_{\pi} S \sigma_{2}$ & $S \sigma_{1}, S \sigma_{2}$ \\
\hline
\end{tabular}

However, a discrimination appears when plane symmetries are considered (Fig. 13, Table 2):

In this more formal and generic approach, the symmetries of the collective modes are clearly brought out, unlike in the reference jet approach. Note that the gearing and simulianeity modes are now differentiated on the basis of distinct plane symmetries, unlike in Fig. 11. Moreover, the two types of collective modes based on either a sinuous or a helical instability mode of the jets can be reconciled into the same approach.

\section{Conclusion}

In this paper, a new experimental set-up is presented where submerged water jets impinge from below at normal incidence on a water/air interface. Above some flow rate threshold value, each jet exhibits a transition and starts oscillating at a well-defined frequency. When the oscillating jets are coupled together in bidimensional lattices, collective phase-locking modes are observed, whose spatial arrangement is similar to that encountercd in crystals. For each geometry (square, hexagonal and triangular), different collective modes are identified and stability diagrams are constructed. The coupling mechanism between the jets is analysed. Surface waves provide the most plausible means of coupling, and a tuning criterion of stability is proposed to explain the bifurcations from one mode to another. The interaction of the oscillating jet with the surface waves emitted by the network is currently under investigation. The symmetries of the system are then investigated using two different approaches. Theoretical permitted modes are predicted that are compared to the observations, and the discrepancies observed between the three geometries are explained. The phase-locking modes observed for the system of coupled water jets are analogous to those encountered in systems of coupled oscillators in Nature, such as in animal locomotion $[14,15]$. The phase ordering observed in the 
collective modes displays no spatial modulation, and all the jets oscillate at the same frequency with the same amplitude. In order to destabilize the system towards spatio-temporal chaos, we propose either to increase the size $N$ of the system and/or the frequency bandwidth $\Delta f$ of the population of oscillators, or to increase the strength of the coupling (testing different liquids) between the jets, as inferred from numierical modelling [4].

\section{Acknowledgements}

We would like to tiank M. Labouise, P. Hède and B. Ozenda for their technical assistance. We also thank M. Bonetti, M. Dubois, L. Laurent and P. Manneville for fruitful discussions.

\section{References}

[1] Y. Kuramoto, Chemical Oscillations, Waves, and Turbulence (Springer, Berlin, 1984).

[2] Y. Kuramoto and I. Nishikawa, J. Statist, Phys. 49 (1987) 569.

|3| K. Kaneko, Physica D 4 I (1990) 137; Physica D 54 (1991) 5.

[4] P.C. Matthews, R.E. Mirollo and S.H. Strogatz, Physica D 52 ( 1991 ) 293.

[5] L. Fubini and K. Wiesenfeld, Phys. Rev. A 43 (1991) 2640.

[6] V. Hakim and W.-J. Rappel, Phys. Rev. A 46 (1992) 7347.

[7] N. Nakagnwa and Y. Kuramoto, Progr. Theoret. Phys. 89 (1993) 313.

[8] H.M. Smith, Science 82 (1935) 151.

[9] M.J. Russell, G.M. Switz and K. Thompson, Pharmacol. Biochem. Behav. 13 (1980) 737.

[10] A.T. Winfree, Scientific American Press (1987).

[11] D.C. Michaels, E.P. Matyas and J. Jalife, Circulation Res. 61 (1987) 704.

[12] J. Buck, Quart. Rev. Biol. 63 (1988) 265.

[13] R.E. Mirollo and S.H. Strogatz, SIAM J. Appl. Math. 50 (1990) 1645 .
[14] J.J. Collins and I.N. Stewart, J. Nonlinear Sci. 3 (1993) 349.

[15] A.T. Winfree, J. Theoret. Biol. 16 (1967) 15.

[16] Y. Kuramoto, in: Int. Symp. on Mathematical Problems in Theoretical Physics. In: H. Araki, ed. (Springer, New York, 1975) 420.

[17] G. Schöner, W.Y. Yiang and J.A.S. Kelso, J. Theoret. Biol. 142 (1990) 359.

[18] P. Hadley, M.R. Beasley and K. Wiesenfeld, Phys. Rev. B 38 (1988) 8712.

[19] P. Tabeling, S. Burkhart, O. Cardoso and H. Willaime, Phys. Rev. Lett. 67 (1991) 3772.

[20] H.-G. Purwins and Ch. Radehaus, in: Neural and Synergetic Computers, ed. H. Haken (Springer, Berlin, 1988).

[21] C.M. Ho and N.S. Nosseir, J. Fluid Mech. 105 (1981) 119.

[22] C.C. Landreth and R.J. Adrain, Exp. Fluids 9 (1990) 74.

[23] J. Stevens and B.W. Webbe, Int. J. Heat Mass Transf. (UK) 36 (1993) 4283.

[24] D.G. Anthony, A. Hirsa and W.W. Willmarth. Phys. Fluids A 3 (1991) 245.

[25] C.K. Madnia and L.P. Bernai, J. Fluid Mech. 261 (1994) 305.

[26] E. Villermaux and E.J. Hopfinger, Physica D 72 (1994) 230.

[27] S. Houard, F. Daviaud and P. Bergé, EuroPhys. Lett. 32 (2) (1995) 101.

[28] E. Guyon, J.-P. Hulin and L. Petit, Hydrodynamique Physique, ed. InterEditions/Editions du CNRS (1991).

[29] G. Batchelor and G. Gill, J. Fluid Mech. 14 (1962) 529.

[30] A.J. Reynolds, J. Fluid Mech. 14 (1962) 552.

[31) G.E. Mattingly and C.C. Chang, J. Fluid Mech. 65 (1974) 541.

[32] M. Bonetti, R. Meynart, J.P. Boon and D. Olivari, Phys. Rev. Lett. 55 (1985) 492.

[33] A. Maurel, Instabilité d'un jet confiné, Thèse de Doctorat de l'Université Paris Vl, France (1994).

[34] M. Lessen and P.J. Singh, J. Fluid Mech. 60 (1973) 433.

[35] P.J. Morris, J. Fluid Mech. 77 (1976) 511.

[36] E. Villermaux, Auto-oscillation tt Mélange dans les écoulements recirculants, Thèse de Doctorat de l'Université Paris VI, France (1993).

[37] M. Golubitsky and I.N. Stewart, Arch. Rational Mech. Anal. 87 (1985) 107. 